\title{
Structural Analysis of the Software Architecture - A Maintenance Assessment Case Study
}

\author{
Catherine Blake Jaktman, John Leaney, and Ming Liu \\ Computer Systems Engineering, Faculty of Engineering, University of Technology, Sydney, \\ Australia \\ \{cjaktman, jleaney,mliu\}@eng.uts.edu.au
}

Key words: Software architecture, software evolution, maintenance measurements, experience report, architectural erosion.

\begin{abstract}
Architectural erosion is a sign of reduced architectural quality. Quality characteristics of an architecture, such as its ability to accommodate change, are critical for an evolving product. The structure of an architecture is said to be eroded when the software within the architecture becomes resistant to change or changes become risky and time consuming. The objective of our work is to understand the signs of architectural erosion that contribute to decreased maintainability. A maintenance assessment case study is described in which we apply structural measurements to a product to determine signs of architectural erosion. It provides an understanding of a product's quality by examining the structure of its architecture. The ability to assess architectural erosion in an evolving software product allows the quality of the architecture to be monitored to ensure its business and maintenance goals are achieved.
\end{abstract}

\section{INTRODUCTION}

\subsection{Software evolution}

Successful software systems experience continual evolution (Lehman 1989) due to events in the system's environment, usage, and business domain. Software systems frequently experience increased maintenance (Parnas 1994) and degradation from continual changes made during software maintenance activities (Bohner 1991). Often, once a system begins to show

The original version of this chapter was revised: The copyright line was incorrect. This has been corrected. The Erratum to this chapter is available at DOI: 10.1007/978-0-387-35563-4_35 
signs of degradation, the maintainability of the system will continue to worsen over time as more software changes are implemented in a system that has grown in size and complexity.

During software evolution, the high-level organisation of the overall system, the software architecture, becomes the critical aspect of design (Garlan 1995; Grisworld and Notkin 1995). The software architecture represents the organisation of a system as a composition of components, connections, and constraints (Garlan 1995). The structure of the architecture includes the gross organisation of the system and global control structure; protocols for communication, synchronisation, and data access; assignment of functionality to design elements; physical distribution; composition of design elements; scaling and performance; and selection among design alternatives (Garlan and Shaw 1993). The structural properties of a software architecture can be expressed in terms of components, interrelationships, principles, and guidelines about their use.

\subsection{Architectural quality}

When the quality of the architecture deteriorates functional adaptations become difficult (Bakker and Hirdes 1995; Kogut and Clements 1998). Additionally, design decisions made at the architectural level have farreaching consequences on the resultant code (Turver and Munro 1994). Certain architectural design decisions may restrict the ability of a software component or interface to be easily modified, or require many components to be modified. Architectural quality is also important for organisations that have evolving product-line architectures. A well-executed and maintained architecture enables organisations to respond quickly to a redefined mission or new and changing markets (Dikel et al. 1997; Morris and Ferguson 1993).

The focus of our work is to understand the signs of reduced architectural quality leading to an increase in maintenance difficulty during the evolution of a product. We want to be able to monitor the maintainability of a product throughout its evolution, to allow functional and non-functional software requirements to be implemented without affecting the following factors

- the flexibility or extendibility of the product

- the understanding of the software architecture

- the maintenance effort required to perform maintenance tasks

\subsection{Architectural erosion}

Our interest in architectural quality is similar to work in architectural erosion and drift by Perry and Wolf (Perry and Wolf 1992). Perry and Wolf define architectural erosion as "violations in the architecture that lead to 
increased system problems and brittleness". They define architectural drift as "a lack of coherence and clarity of form which may lead to architectural violation and increased inadaptability of the architecture". Their work mentions the importance of the architecture's style to encapsulate decisions about the architectural elements, constraints and relationships that are needed to understand architectural violations such as erosion and drift.

We have extended Perry and Wolf's definition of erosion to include the structure of an architecture. We define the structure of a software architecture to be eroded when the software within the architecture becomes resistant to change or software changes become risky and time consuming. Erosion can also be exhibited when the software is hard to understand or manage due to an increase in the size and complexity of the code and its structure. Erosion can be a result of poor design decisions made whilst implementing maintenance changes to the system, or a result of limited architectural understanding during software maintenance that may have constrained the flexibility of the design.

\subsection{Characteristics of erosion}

The characteristics of architectural erosion in an evolving product are listed below in Table 1 .

Table 1: Characteristics of erosion

The complexity of the architecture has increased from a previous release as shown by an increase in the structural complexity measurements.

The impact of a software modification results in unpredictable software behaviour (e.g., ripple effect).

The architecture is not documented or its structure is not explicitly known.

The relationship between the architectural representation and the code is unclear or hard to understand.

There is a continual increase in the defect rate that is disproportionate to the amount or type of maintenance work performed (e.g., new functionality added or technology upgrades).

Greater resources are required to implement a software change (i.e. understand, code and test).

Experience with the software becomes crucial to understanding how to implement a software change.

Certain software changes may become too risky or costly to implement.

The design principles of the architecture are violated when implementing a product variant (e.g., code redundancy due to cloning).

The system may become resistant to change (i.e. "brittle"), or require additional operational procedures (e.g., manual tasks) to support new functionality. 


\subsection{Structural signs of erosion}

The signs of architectural erosion are determined by studying the changes in the size and complexity of the product's architecture. If the results of the measurements change in such a way to indicate increased maintainability (i.e., increased complexity), the architecture is studied further to determine if the measurements indicate the presence of erosion. The signs of erosion are validated by the software developers to ensure agreement that the software has eroded. In the long term this may be developed as a sensitive indicator of impending erosion, and the architectural quality preserved.

A description of the architectural measurements we have chosen to help us understand architectural erosion are included in sections 2.4, 2.5, and 2.6 of this paper. The measurements were chosen on the basis of their history of usage and understanding, and their ready availability.

\section{MAINTENANCE ASSESSMENT CASE STUDY}

This section describes the framework of the maintenance assessment case study. The objective of the maintenance assessment case study is to identify useful measurements that will allow us to determine the signs of architectural erosion in an evolving product.

\subsection{Approach}

In the maintenance assessment case study we apply structural measurements to the architectural properties of a product. The measurements are applied to each operational release of the product throughout the evolution of the product. They are generated using the Logiscope $^{T M}$ code analysis tool (Logiscope 1997-1998). The measurements between each release are compared and analysed to detect signs of architectural erosion. The steps in the maintenance assessment approach are shown in Table 2.

\section{Table 2: Case study process}

Step 1: Select an architectural viewpoint for analysis of the system.

- Criterion: the architectural viewpoint should conform to some accepted viewpoint

Step 2: Take measures on selected releases of the product.

- Criterion: structural measures selected should conform to recognised measures

- Criterion: selected measures should be fair indicators of structural erosion as described in sections 1.3 Architectural erosion and 1.4 Characteristics of erosion

- Criterion: the selected releases should give a fair view of the product 
Step 3: Analyse the structural measures for change.

- Criterion: change should be such as to be attributable to erosion

- Criterion: change should be defined as outside some expected 'noise' values

Step 4: Interpret the measures in terms of (structural) erosion.

- Criterion: interpretation should be consistent with structural erosion as described in sections 1.3 Architectural erosion and 1.4 Characteristics of erosion

Step 5: Translate the structural erosion conclusions into (maintenance) programming terms.

- Criterion: use documented maintenance programming practice terms

Step 6: Validate with project maintenance programmers (interviewees)

- Criterion: ensure the experiment is blind, i.e. the interviewees do not know of the analysis before the interpretation is complete.

- Criterion: ensure terms are fully understood by interviewees

- Criterion: use accepted techniques such as questionnaires

- Criterion: ensure interviewees have 'last word' on conclusions

\subsection{Proposed measures}

The proposed measures used to determine architectural erosion include:

- general measures

- basic architectural measures

- derived architectural measures

\subsection{General measures}

The general measures listed in Table 3 are taken for each selected software release of the product. The general product measures are used to understand the age and growth in the size of the product.

\begin{tabular}{|c|}
\hline Version No. \\
\hline Date of Release \\
\hline Lines of Code \\
\hline No. of Runtime Files \\
\hline Total Components \\
\hline
\end{tabular}

\subsection{Basic architectural measures}

Architectural measures are taken to provide an understanding of the overall view of the system. Architectural measures are based on the call 
graph representation of the product and represent the complexity of the structure of the architecture. The basic architectural measures (or counts) are defined in Table 4.

Table 4. Basic architectural measures

\begin{tabular}{ll}
\hline $\begin{array}{l}\text { Components (or Nodes) } \\
\text { Number of Components (or Nodes) }\end{array}$ & $\begin{array}{l}\text { Number of components (or nodes) in the relative } \\
\text { call graph. An architectural-level component } \\
\text { represents a source code file. }\end{array}$ \\
\hline $\begin{array}{l}\text { Edges } \\
\text { Number of Edges }\end{array}$ & $\begin{array}{l}\text { Number of calls between components. This is } \\
\text { the same as edges in the relative call graph. A } \\
\text { relative call graph is a diagram that identifies the } \\
\text { components in a system and shows which } \\
\text { modules call one another. }\end{array}$ \\
\hline $\begin{array}{l}\text { Call Paths } \\
\text { Number of Relative Call Graph Call- }\end{array}$ & $\begin{array}{l}\text { Number of calling paths in the relative call graph, } \\
\text { from the component to each leaf components in } \\
\text { the graph. }\end{array}$ \\
\hline $\begin{array}{l}\text { Levels } \\
\text { Number of Relative Call Graph }\end{array}$ & Number of hierarchical levels in the relative call \\
Levels & graph. \\
\hline
\end{tabular}

\subsection{Derived architectural measures}

The derived measurements taken at the architecture-level (based on the "counts" of Table 4) are defined and explained in Table 5. The measurements listed in Table 5 are supported in the Logiscope ${ }^{\mathrm{TM}}$ code analysis tool (Logiscope 1993).

Table 5: Derived architectural measures

\begin{tabular}{ll}
\hline Hierarchical Complexity & Average number of components per relative call graph \\
Relative Call Graph & level (number of components divided by number of \\
Hierarchical Complexity & levels). \\
\hline $\begin{array}{l}\text { Structural Complexity } \\
\text { Relative Call Graph }\end{array}$ & $\begin{array}{l}\text { Average number of calls per component (number of } \\
\text { complexity }\end{array}$ \\
\hline Average Components/Path & Average number of components per call path \\
& (components divided by call paths) \\
\hline Average Paths/Component & Average number of paths per component (call paths \\
& divided by the number of components.) \\
\hline
\end{tabular}




\section{THE CASE STUDY}

\subsection{The Squid product}

In the case study we applied the aforementioned measurements to the Squid product (Squid), which was developed at the National Laboratory for Advanced Networking Research (NLANR) at the University of California at San Diego. Squid is an Internet object caching product. The Squid product was chosen as historical data was publicly available for each operational release of the product throughout its evolution. The Squid product is written in the $\mathrm{C}$ programming language and is implemented in the Unix operating environment. Six releases of Squid spaced approximately at equal time intervals were chosen for analysis.

\subsection{Approach}

In our approach structural measurements are taken for each release of the Squid product. We then interpret the measurements, and put the interpretations to the Squid developers to see if our interpretation of the measures made any sense to them. (Being our initial case study, there were no hypotheses developed. The purpose of this work was to see if we could find any encouragement for using existing structural measurements for this research problem.) The steps followed in the case study are outlined in Table 2; the sub-headings below follow the steps outlined in the table.

\subsubsection{Step 1: Select architectural viewpoint}

The ideal architectural viewpoint would be to work with the subsystems defined by the original software developers, and understand changes in those subsystems and the relationships between them. This would be in accord with the software architecture definition whereby one represents the organisation of a system as a composition of components, connections and constraints (Garlan 1995).

From this perspective, Squid consists of the following major subsystems:

- the Client Side (icp.c, client_side.c)

- the Server Side (proto.c, http.c, ftp.c, gopher.c, wais,c, ssl.c, pass.c)

- the Storage Manager (store.c)

- other sub-systems, including Neighbours; IP/FQDN Cache; DNS

Servers; Cache Manager; Network Probe Database; Redirectors and Access Controls. 
Unfortunately, to quote the Squid developers, "the Squid source code has evolved more from empirical observation and development rather than a solid design process over two years or more. It carries a legacy of being 'touched' by numerous individuals, each with somewhat different techniques and terminology" (Wessels 1997).

We have created an architectural view based on "the view from the top". The architecture viewpoint, which appears repeatable for procedural languages such as $\mathrm{C}$, is to choose the 'main' routine as a reference node for structural measures. Our structural measures are based on the call graph, and are taken from the main routine alone. Thus, we will only include components that are called on a path from main.

\subsubsection{Step 2: Take measures of selected releases}

In this step the general, architectural, and derived architectural measures are taken on the selected releases of the product.

\subsubsection{Squid - general measures}

The general measures for Squid, as specified in section 2.3 General measures, are given in Table 6 below.

Table 6: Squid general measurements

\begin{tabular}{lcccccc}
\hline Version No. & V1.0.0 & V1.0.22 & V1.1.0 & V1.1.10 & V1.1.14 & V1.1.21 \\
\hline Date of Release & Jul. 1996 & Oct. 1996 & Dec. 1996 & Apr. 1996 & Jul. 1997 & Apr. 1998 \\
\hline No. of Files & 32 & 33 & 42 & 44 & 44 & 44 \\
\hline Lines of C Code & 23587 & 24996 & 27425 & 30008 & 30222 & 29329 \\
\hline Total Components & 619 & 630 & 693 & 733 & 739 & 748 \\
\hline
\end{tabular}

\subsubsection{Squid - architectural measures}

The architectural measures for Squid, as specified in 2.4 Basic architectural measures are given in Table 7 below. The viewpoint is from the main routine. The reduction in the number of nodes and edges accords with the reduction in the lines of code in Table 6.

Table 7: Squid basic architectural measures (counts)

\begin{tabular}{lcccccc}
\hline Version No. & V1.0.0. & V1.0.22 & V1.1.0 & V1.1.10 & V1.1.14 & V1.1.21 \\
\hline Levels & 18 & 21 & 23 & 19 & 21 & 20 \\
\hline Nodes & 413 & 421 & 416 & 429 & 438 & 434 \\
\hline Edges & 1243 & 1267 & 1319 & 1399 & 1428 & 1415 \\
\hline Call Paths & 24264 & 28037 & 32784 & 40616 & 80848 & 80952 \\
\hline
\end{tabular}




\subsubsection{Squid - derived architectural measures}

The derived architectural measures for Squid, as specified in 2.6 Derived Basic architectural measures are specified in Table 8 below.

Table 8: Squid derived architectural measures

\begin{tabular}{lcccccc}
\hline Version No. & V1.0.0 & V1.0.22 & V1.1.0 & V1.1.10 & V1.1.14 & V1.1.21 \\
\hline Call Paths & 24264 & 28037 & 32784 & 40616 & 80848 & 80952 \\
\hline Levels & 18 & 21 & 23 & 19 & 21 & 20 \\
\hline Hierarchical Complexity & 22.94 & 20.04 & 18.08 & 22.57 & 20.85 & 21.70 \\
\hline Structural Complexity & 3.01 & 3.01 & 3.17 & 3.26 & 3.26 & 3.26 \\
\hline Average Paths/Component & 58.75 & 66.59 & 78.8 & 94.67 & 184.58 & 186.52 \\
\hline Average Components/Path & 0.01 & 0.01 & 0.01 & 0.01 & 0 & 0 \\
\hline
\end{tabular}

\subsubsection{Step 3: Analyse the structural measures for change}

The major changes that occurred, and the discussion, are shown in Table 9. Other changes were considered to be too dubious to discuss. Considering all the data presented to date, a strong observation can be made that the system started to stabilise at V1.1.0. This is shown by a slower growth rate in the size of the product and a reduced rise in the complexity of the product. Therefore, we will only comment on changes after V1.1.0.

Table 9: Analysis of changes in Squid

\begin{tabular}{ll}
\hline Property & \multicolumn{1}{c}{ Discussion } \\
\hline Components & $\begin{array}{l}\text { The number of components has been practically constant throughout the } \\
\text { life of the project. }\end{array}$ \\
\hline Call paths & $\begin{array}{l}\text { The number of call paths is arguably high compared with } \\
\text { recommendations in (Logiscope 1993) even from the beginning of the } \\
\text { project. The number of call paths has changed by a factor of (about) } 3.5 \\
\text { during the life of the project, with a sharp rise between V1.1.10 and }\end{array}$ \\
& V1.1.14 (as shown in Table 7). \\
\hline Component & $\begin{array}{l}\text { The components were analysed using the ISO 9126 maintainability } \\
\text { maintainability } \\
\end{array}$ \\
& $\begin{array}{l}\text { measures (ISO 1991). The component maintainability for Squid was } \\
\text { consistent thought the product evolution (i.e. 2\% excellent, 89\% good, } \\
4 \% \text { fair, 3\% poor and 2\% undefined). }\end{array}$ \\
\hline
\end{tabular}

\subsubsection{Steps 4 and 5: Interpretation and translation}

These steps involve interpreting the measures in terms of (structural) erosion and translating the structural erosion conclusions into (maintenance) programming terms. The analysis of changes in Squid (Table 9) suggests 
that with the increase in call paths, and the number of components remaining the same, that the changed (or new) components will have become much harder to integrate with each release. However, the small change to component maintainability suggests that changes to the component itself will not be hard.

\subsubsection{Step 6: Validate with project maintenance programmers (interviewees).}

A basic questionnaire was developed based on the conclusions of Step 4: Interpret the measures in terms of (structural) erosion, and Step 5: Translate the structural erosion conclusions into (maintenance) programming terms. This was sent (via e-mail) to the Squid software team leader, in order to get a response from a maintenance programmer. The reply was then analysed, and interpreted back as a second series of questions. A final conclusion was then drawn.

Based on this interpretation, the following statement was put to the Squid programmers:

"Our conclusion would be that individual modules in
Squid are OK to maintain, but integration of the modules
into the Squid system can at times be difficult."

The first response to this statement included a lack of match between the statement and the Squid system. This is exemplified by the two responses, "I do not think there is a good notion of modularity in Squid." and "Thus, I cannot answer your question since I do not see a lot of well-defined "modules" in Squid." In the light of these responses, we sent another set of questions:

Our statement: Typically, the extra load of a module (doing $\mathrm{Y}$ and $\mathrm{Z}$ as well as $\mathrm{X}$ in your email) occurs as a result of change. Side effects can also occur as a result of change, or simply wishing to get the job done ASAP.

Squid response: This is certainly true, but, from what I know, certain "extra load" was there from the very beginning of Squid. Also note that HTTP itself ..... often forces programmers to violate many good software engineering design principles.

Our statement: You attempt to modify some part of Squid. As a result you find that a particular collection of code (an approximate module) is the most likely place to start. 
Squid response: OK. Although any serious change would require modification in several files/modules.

Our statement: You then find that a piece of code does other things as well, so you have to be careful to change only what you want to change. And then there is the question of side effects. So I relate that to being able to change parts of code, but finding it more difficult to get Squid running properly again.

Squid response: I somewhat agree with your last statement. You see, when I modify a part of the code, I usually know a priori that I will have to modify other places. Thus, if somebody asks me a question like "is it easy to add feature W?", I reply based on the total amount of modifications that I foresee, and not based on the first change that I will make. In other words, most significant changes will require modification of several "modules", which is hard. And nobody cares about minor updates, I guess.

\subsection{Conclusion from the interviews}

If we now return to our original statement, there seems to be considerable reinforcement for the proposition that integration of modules into Squid is difficult. But, it is not obvious that individual modules are easy to maintain from the interview discussion. We can determine that the component level maintenance measures (ISO 9126) will not reflect the increased complexity that is shown at the architectural level (as indicated by the call path measure).

The problem of software integration is due to multiple functional modules and side effects. This is supported by the increase in call paths, with little increase in the number of components. The problem of side effects could possibly have been seen by the decrease in the number of comments, as the system became older and more difficult to understand.

\subsection{Summary and conclusions}

In Table 10, a summary of the characteristics of erosion are matched with the measurements and interviews from the Squid software team.

There is some promise in using structural measures to predict erosion. This has been an exploratory case study, but nevertheless the ability to predict some of the features of the Squid system are most encouraging. 
Table 10: Characteristics of erosion matched with measures and interviews

\begin{tabular}{|c|c|c|}
\hline Characteristic & Measures & Interviews \\
\hline $\begin{array}{l}\text { The complexity of the architecture } \\
\text { has increased from a previous } \\
\text { release as shown by an increase in } \\
\text { the structural complexity } \\
\text { measurements. }\end{array}$ & $\begin{array}{l}\text { Shown in structural } \\
\text { measures (call paths). }\end{array}$ & $\begin{array}{l}\text { Not validated, we don't } \\
\text { have coverage of } \\
\text { enough releases, yet. }\end{array}$ \\
\hline $\begin{array}{l}\text { The impact of a software } \\
\text { modification results in } \\
\text { unpredictable software behaviour } \\
\text { (e.g., ripple effect). }\end{array}$ & $\begin{array}{l}\text { Shown in structural } \\
\text { measures (call paths). }\end{array}$ & Validated \\
\hline $\begin{array}{l}\text { The architecture is not documented } \\
\text { or its structure is not explicitly } \\
\text { known. }\end{array}$ & $\begin{array}{l}\text { No documentation of the } \\
\text { architecture was } \\
\text { available. }\end{array}$ & Validated \\
\hline $\begin{array}{l}\text { The relationship between the } \\
\text { architectural representation and the } \\
\text { code is unclear or hard to } \\
\text { understand. }\end{array}$ & $\begin{array}{l}\text { No documentation of the } \\
\text { architecture was } \\
\text { available. }\end{array}$ & Validated \\
\hline $\begin{array}{l}\text { There is a continual increase in the } \\
\text { defect rate that is disproportionate } \\
\text { to the amount or type of } \\
\text { maintenance work performed (e.g., } \\
\text { new functionality added or } \\
\text { technology upgrade). }\end{array}$ & $\begin{array}{l}\text { Unknown } \\
\text { defect rate data not } \\
\text { captured. }\end{array}$ & Not validated \\
\hline $\begin{array}{l}\text { Greater resources are required to } \\
\text { implement a software change (i.e. } \\
\text { understand, code and test). }\end{array}$ & $\begin{array}{l}\text { Unknown } \\
\text { data not captured. }\end{array}$ & Not validated \\
\hline $\begin{array}{l}\text { Experience with the software } \\
\text { becomes crucial to understanding } \\
\text { how to implement a software } \\
\text { change. }\end{array}$ & $\begin{array}{l}\text { Suggested by structural } \\
\text { measures (call paths). }\end{array}$ & Validated \\
\hline $\begin{array}{l}\text { Certain software changes may } \\
\text { become too risky or costly to } \\
\text { make. }\end{array}$ & $\begin{array}{l}\text { Unknown data not } \\
\text { captured. }\end{array}$ & Not validated \\
\hline $\begin{array}{l}\text { The design principles of the } \\
\text { architecture are violated when } \\
\text { implementing a product variant } \\
\text { (e.g., code redundancy due to } \\
\text { cloning). }\end{array}$ & $\begin{array}{l}\text { Unknown data not } \\
\text { captured, although can } \\
\text { be determine based on } \\
\text { changes in architectural } \\
\text { views and component } \\
\text { usage. }\end{array}$ & Not validated \\
\hline $\begin{array}{l}\text { The system may become resistant } \\
\text { to change (i.e. "brittle") or requires } \\
\text { additional operational procedures } \\
\text { (e.g., manual tasks) to support new } \\
\text { functionality. }\end{array}$ & $\begin{array}{l}\text { Unknown data not } \\
\text { captured. May not be } \\
\text { displayed (yet) in a } \\
\text { system that is only } 2 \\
\text { years old. }\end{array}$ & Not validated \\
\hline
\end{tabular}

Our study showed that in order to understand the quality of the architecture one must look at the component measures, as well as the 
structural architectural measures, in order to ascertain the origin of change in the structure of the architecture. For example, changes to the structure could occur as a result of a redesign that gives rise to the addition of components, thus changing the connections. Alternatively, modifications to components can affect the structure through additional calls to other components, and side effects. In the case of the Squid system, very little happened to the structure through redesign, as shown by the relatively constant number of components. However, the modules were changed to give new functionality (as described in the interviews), this is shown by the increase in the number of call paths. However, it is interesting that the number of call paths was high from the beginning, suggesting an early flaw in the architecture.

\section{RELATED WORK}

The most extensive software evolution study has been the FEAST project which started in 1997 (Lehman et al. 1997). This work and subsequent work on the FEAST project formed the foundation for describing the laws of software evolution and the properties of a E-type system. In this work the size of modules were used to understand the growth rate and change rate of the system, and its affect on software quality. Additional software evolution work that applied metrics included projects to determine; code degradation (Ash et al. 1994), entropy (Coleman 1995; Harrison and Cook 1990) and erosion (Kogut and Clements 1994). The focus of such work is at the program code level, using measurements such as LOC, Halstead, and McCabe, to determine the complexity of the program that may be errorprone or change-prone.

However, quantitative approaches to understanding architectural quality have been limited. The evolution of a telecommunications system has been studied to identify modules or sub-systems in the architecture that should be considered for restructuring or re-engineering (Gall et al. 1997). In their work they considered the size of each system, the change rate and growth rate. The complexity of the architecture has also been measured using pattern coverage techniques: the proportion of an architecture that can be covered by patterns and the number of patterns it takes to cover the architecture (Kazman and Burth 1998). These are complementary measures of the system's regularity, and hence its architectural complexity. These quantitative approaches differ from our work as their focus in on identifying weak areas of the architecture for re-engineering. Our work uses structural measurements to determine the maintainability of the architecture to support the evolution of the product. 
Qualitative techniques such as the Software Architecture Analysis Method (SAAM) (Kazman et al. 1994), and the AQA (Architecture Quality Assessment) (Hilliard, Kurland, and Litvintchouk 1997) provide a method to gain information about a system's qualities (e.g., modifiability, security, portability). Such techniques can be used for multiple purposes such as, to consider future changes to the system or how an architecture will accommodate change. However, the qualitative architecture evaluation techniques do not clearly state a criterion for maintainability that can be measured and verified. Additionally, architectural evaluation techniques are limited in providing an understanding of the signs and causes of reduced quality.

\section{BENEFITS}

The maintenance assessment case study provides preliminary work in determining a set of measures that can be applied to understand the quality of a software architecture during its evolution. The ability to identify signs of architectural erosion allows us to make improvements to the architecture to increase its flexibility and longevity prior to reaching further erosion. An architectural maintenance assessment method can also be used to

- derive quality benchmarks that are necessary to evolve the product; and

- build knowledge about the factors that influence the quality of the product-line architecture.

This work can also provide insights into the causes of erosion, allowing us to improve architectural analysis and design techniques in the early phases of system development. For example, proven guidelines for assessing the extendibility and flexibility of an architecture will allow us to assess the adaptability of an architecture using pre-design data (e.g., module hierarchy).

\section{FUTURE WORK}

We would like to continue our research with the Squid product to gather measurements on additional software releases of the system as the product evolves. Additionally, we would like to apply this research to a system that is older and larger; this would allow us to study differences in the results that may be due to varying organisational, software process, and architectural design factors. 


\section{REFERENCES}

Ash, D., Alderete, J., Yao, L., Oman, P. W., and Lowther, B. 1994. "Using Software Maintainability Models to Track Code Health", Proceedings of the 1994 International Conference on Software Maintenance. pp. 154-160.

Bakker, G., and Hirdes, F. 1995. "Recent Industrial Experiences with Software Product Metrics". Proceedings of the Second Symposium on Software Quality Techniques and Acquisition Criteria. Florence, Italy. pp. 179-191.

Bohner, S. A. 1991. "Software Change Impact Analysis for Design Evolution", Proceedings of the $8^{\text {th }}$ Int'l, Conference on Software Maintenance and Re-engineering. IEEE CS Press., Los Alamitos. pp. 292-301.

Coleman, D., Ash, D., Lowther, B., and Oman, P. 1995. "The Application of Software Maintainability Models in Industrial Software Systems". Journal of Systems and Software. 29:3-16.

Dikel, D., Kane, D., Ornburn, S., Loftus, B., and Wilson, J. 1997. “Applying Software Product-Line Architecture". IEEE Software. 30:49-55.

Gall, H., Jazayeri, M., Klösch, R., and Trausmuth, G. 1997. "Software Evolution Observations Based on Product Release History", Proceedings of the International Conference on Software Maintenance, Bari, Italy, October. pp. 160-166.

Garlan, D. 1995. "First International Workshop on Architectures of Software Systems Workshop Summary". ACM SIGSOFT, Software Engineering Notes. 20:3:84-89.

Garlan, D., and Shaw, M. 1993. An Introduction to Software Architecture, Advances in Software Engineering and Knowledge Engineering, Vol I, River Edge, NJ:World Scientific Publishing Company.

Grisworld, W. G., and Notkin, D. 1995. "Introduction to the Special Issue on Software Architecture", IEEE Transactions on Software Engineering. (April) 21:9-287.

Harrison, W., and Cook, C. 1990. "Insights on Improving the Maintenance Process Through Software Measurement". Proceedings of the Conference on Software Maintenance. San Diego, CA., pp. 37-45.

Hilliard III, R., F., Kurland, M. J., and Litvintchouk, S. D. 1997. Mitre's Architecture Quality Assessment. Software Engineering \& Economics Conference.

ISO 1991. International Standard ISO/IEC 9126. Information Technology Software Product Evaluation - Quality characteristics and guide-lines for their use, International Organization for Standardization, International Electrotechnical Commission, Geneva.

Kazman, R., Bass, L., Abowd, G., and Webb, M. 1994. "SAAM: A Method for Analyzing the Properties of Software Architectures". Proceedings of the 16th International Conference on Software Engineering, Sorrento, Italy.

Kazman, R., and Burth, M. 1998. "Assessing Architectural Complexity". Proceedings of the $2^{\text {nd }}$ Euromicro Working Conference on Software Maintenance and Re-engineering (CSMR'98). IEEE Computer Society Press.

Kogut, P., and Clements, P. 1994. "The Software Architecture Renaissance", Crosstalk, The Journal of Defense Software Engineering.7:20-24.

Lanubile, F., and Visaggio, G. 1992. "Software Maintenance by Using Quality Levels", Workshop on Software Quality: Measurement and Practice.

Lehman, M. M. 1989. "Uncertainty in Computer Application and its Control Through the Engineering of Software". Software Maintenance: Research and Practice. 1:3-27.

Lehman, M.M., Ramil, J. F., Wernick P.D., Perry, D. E., and Turski, W. M. 1997. "Metrics and the Laws of Software Evolution - the Nineties View". Proceedings of the Fourth Software Metrics Symposium. pp. 20- 32.

Logiscope ${ }^{\mathrm{TM}}$ Editor Manual, 1993. 
Logiscope $^{\mathrm{TM}}$ by Verilog, Version 2.1, 1997-1998. Supported by Prophecy Technology in Australia, URL: http://www.prophecy.com.au

Morris, C. R., and Ferguson, C. H. 1993. "How Architecture Wins Technology Wars", Harvard Business Review. pp. 86-96.

Parnas, D. L. 1994. "Software Aging. In Proceedings of $16^{\text {th }}$ International Conference on Software Engineering (ICSE 16), Sorrento, Italy. pp. 279-298.

Perry, D. E., and Wolf, A. L. 1992. "Foundations for the Study of Software Architecture". ACM SIGSOFT Software Engineering Notes. 17:4:40-52.

Squid Internet Object Cache, Source code distributions URL: http://squid.nlanr.net/

Turver, R. J., and Munro, M. 1994. "An Early Impact Analysis Technique for Software Maintenance". Journal of Software Maintenance: Research and Practice. 6:35-52.

Wessels, D. and Squid developers, 1997. Squid Programmers Guide (Draft). 